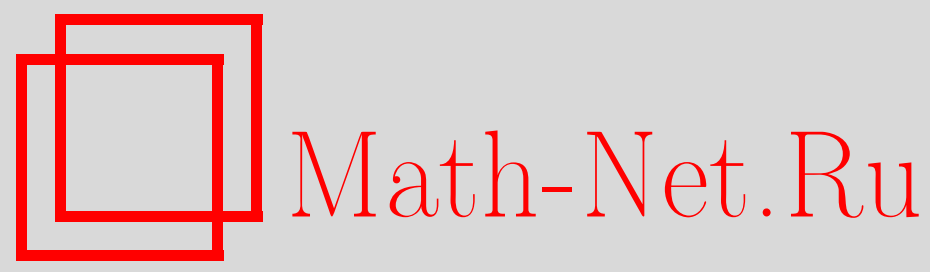

А. В. Мироненко, Равномерное приближение классом функций с ограниченной производной, Матем. заметки, 2003, том 74, выпуск 5, 696-712

DOI: https://doi.org/10.4213/mzm302

Использование Общероссийского математического портала Math-Net.Ru подразумевает, что вы прочитали и согласны с пользовательским соглашением http://www . mathnet.ru/rus/agreement

Параметры загрузки:

IP: 34.227 .88 .159

26 апреля 2023 г., 03:08:07

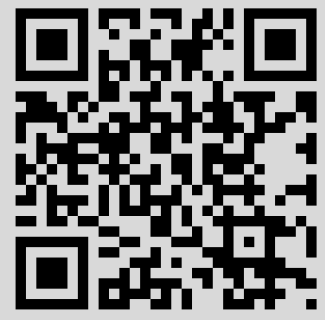




\section{РАВНОМЕРНОЕ ПРИБЛИЖЕНИЕ КЛАССОМ ФУНКЦИЙ С ОГРАНИЧЕННОЙ ПРОИЗВОДНОЙ}

\section{А. В. Мироненко}

В работе рассмотрен вопрос о равномерном приближении заданной на отрезке непрерывной функции. Приближение производится при помощи функции с абсолютно непрерывной производной порядка $n-1$ и ограниченной по модулю производной порядка $n$. Приводится критерий альтернансного типа для элемента наилучшего приближения в этом классе. Данный критерий обобщает полученный Н. П. Корнейчуком критерий элемента наилучшего приближения в классе липшицевых функций.

Библиограффия: 4 названия.

1. Введение. Пусть $C[a, b]$ - это пространство непрерьвных на отрезке $[a, b]$ функций с нормой

$$
\|f\|=\|f\|_{C[a, b]}=\max \{|f(x)|: x \in[a, b]\} .
$$

Пусть $A C^{n-1}[a, b]$ - класс функций, заданных на отрезке $[a, b]$ и имеющих абсолютно непрерьвную производную порядка $n-1$. Через $\mathscr{D}^{n} \subset A C^{n-1}[a, b]$ обозначим подкласс функций, имеющих ограниченную единицей производную порядка $n$, т.е.

$$
\mathscr{D}^{n}=\left\{g \in A C^{(n-1)}[a, b]:\left|g^{(n)}(x)\right| \leqslant 1 \text { всюду, где существует } g^{(n)}(x)\right\} .
$$

Зафиксируем произвольную непрерьвную функцию $f \in C[a, b]$. Назовем $g_{*}$ әлементом наилучшего приближения (ЭНП) для функции $f$ в классе $\mathscr{D}^{n}$, если вьполняется равенство

$$
\left\|f-g_{*}\right\|=\min \left\{\|f-g\|: g \in \mathscr{D}^{n}\right\} .
$$

Задачу о характеризации таких элементов наилучшего приближения при $n=1$ рассматривал Корнейчук [1, с. 219, 220]. При получении точной константы в теореме Джексона-Стечкина он приближал непрерьвные функции при помощи функций с ограниченной константой Липшица:

$$
g \in \operatorname{Lip} 1 \Longleftrightarrow\left|g\left(x_{2}\right)-g\left(x_{1}\right)\right| \leqslant\left|x_{2}-x_{1}\right| \quad \forall x_{1}, x_{2} .
$$

Нетрудно показать, что Lip $1=\mathscr{D}^{1}$. Корнейчуком была указана характеризация ближайших элементов в классе $\mathscr{D}^{1}$ с использованием понятия “жесткого звена", т.е. такого подмножества отрезка $[a, b]$, на котором решение аналогичной локальной задачи дает единственньй ЭНП, и величина наилучшего приближения совпадает с величиной наилучшего приближения в задаче (1). Основной целью данной работы является обобщение результата Корнейчука на случай $n>2$.

Работа выполнена при поддержке Российского фонда фундаментальных исследований, грант № 02-01-00782. 
2. Формулировка результата. Пусть приближаемая функция $f \in C[a, b]-$ фиксирована.

ОПРЕДЕЛЕНИЕ. Набор точек $x_{1}<x_{2}<\cdots<x_{k}$ из отрезка $[a, b]$ назовем $а л ь т е р$ нансом для функции $g \in \mathscr{D}^{n}$, если

$$
(f-g)\left(x_{i}\right)=(-1)^{i+1} \cdot \operatorname{sign}\left((f-g)\left(x_{1}\right)\right) \cdot\|f-g\|
$$

при $i=1, \ldots, k$.

Теорема 1 (Н. П. Корнейчук). Пусть $f \in C[a, b] \backslash \mathscr{D}^{1}$. Для того, чтобы функиия $g_{*} \in \mathscr{D}^{1}$ была ЭНП для функиии $f$ необходимо и достаточно, чтобы на отрезке $[a, b]$ нашелся альтернанс из двух точек $x_{1}<x_{2}$ такой, что

$$
\left.g_{*}^{\prime}\right|_{\left(x_{1}, x_{2}\right)} \equiv-\operatorname{sign}\left(\left(f-g_{*}\right)\left(x_{1}\right)\right) \text {. }
$$

Основным результатом данной статьи является теорема 2 , которая обобщает теорему Корнейчука.

Tеорема 2. Пусть $f \in C[a, b] \backslash \mathscr{D}^{n}$, где $n=1,2, \ldots$ Для того, чтобы функиия $g_{*} \in \mathscr{D}^{n}$ была ЭНП для функиии $f$, необходимо и достаточно, чтобы на отрезке $[a, b]$ нашелся набор точек $t_{0}<t_{1}<\cdots<t_{s}$ и на отрезке $\left[t_{0}, t_{s}\right]$ нашелся альтернанс из $k$ точек $x_{1}<x_{2}<\cdots<x_{k}$ такие, что выполнились бы следующие условия:

1) верны тождества

$$
\left.g_{*}^{(n)}\right|_{\left(t_{i}, t_{i+1}\right)} \equiv(-1)^{n+i} \operatorname{sign}\left(\left(f-g_{*}\right)\left(x_{1}\right)\right) \quad \text { для всех } i \in 0, \ldots, s-1 ;
$$

2) $k \geqslant s+n$.

Альтернанс, удовлетворяющий всем условиям теоремы 2, для краткости мы будем назьвать жестким звеном. Отметим, что при $n=1$ из теоремы 2 следует теорема Корнейчука, а при $n=2$ она приобретает более простой вид.

Tеорема 3. Пусть $f \in C[a, b] \backslash \mathscr{D}^{2}$. Для того, чтобы функиия $g_{*} \in \mathscr{D}^{2}$ была ЭНП для функиии $f$, необходимо и достаточно, чтобы на $[a, b]$ нашелся набор точек $t_{0}<t_{1}<\cdots<t_{s}$ и на отрезке $\left[t_{0}, t_{s}\right]$ нашелся альтернанс из $s+2$ точек $\left\{x_{j}\right\}_{j=1}^{s+2}$ такие, что выполнились бы следующ, уе условия:

1) верны тохдества $\left.g_{*}^{\prime \prime}\right|_{\left(t_{i}, t_{i+1}\right)} \equiv(-1)^{i} \operatorname{sign}\left(\left(f-g_{*}\right)\left(x_{1}\right)\right)$ при $i \in 0, \ldots, s-1$;

2) справедливы включения $x_{1}, x_{2} \in\left[t_{0}, t_{1}\right), x_{s+1}, x_{s+2} \in\left(t_{s-1}, t_{s}\right]$;

3) при $i \in 3, \ldots, s$ верны включения $x_{i} \in\left(t_{i-2}, t_{i-1}\right)$.

\section{3. Доказательство.}

3.1. Обозначения и определения. Через $Q_{+1}(g)=\{x:(f-g)(x)=\|f-g\|\}$ и $Q_{-1}(g)=\{x:(f-g)(x)=-\|f-g\|\}$ будут обозначаться множества точек максимального положительного и отрищательного уклонения функции $g \in \mathscr{D}^{n}$ от функции $f$. Поскольку функции $f$ и $g$ непрерывны, хотя бы одно из них не пусто. Кроме того, существует константа $\gamma=\gamma(g)>0$ такая, что

$$
\forall x \in Q_{+1}(g) \quad \forall y \in Q_{-1}(g) \quad|x-y|>\gamma .
$$


ОПРЕДЕЛЕНИЕ. Пусть функция $g \in \mathscr{D}^{n}$. Отрезок $[c, d] \subseteq[a, b]$ будем назьвать “+"отрезком, если на нем нет точек из $Q_{-1}(g)$, и “-"-отрезком, если на нем нет точек из $Q+1(g)$.

Для краткости будем называть максимальные по включению альтернансы просто максимальными.

ОПРЕДЕЛЕНИЕ. Расширенный альтернанс на отрезке $[c, d] \subseteq[a, b]$ для функции $g \in$ $\mathscr{D}^{n}$ есть набор множеств $\left\{X_{i}\right\}_{i=1}^{k}$ таких, что каждое множество $X_{i}$ состоит из всех $i$-х по счету точек в максимальных альтернансах на отрезке $[c, d]$.

Через $\overline{X_{i}}=\operatorname{conv} X_{i}$ будем обозначать выпуклую оболочку множества $X_{i}$. Для краткости будем говорить, что $y<X_{i}$, если неравенство $y<x$ верно для всех $x \in X_{i}$. Тогда всегда есть точка $z_{i}$ такая, что $X_{i}<z_{i}<X_{i+1}$ и $(f-g)\left(z_{i}\right)=0$.

Будем говорить, что набор точек $\left\{x_{i}\right\}$ является упорядоченным, если для любого $i$ выполняется неравенство $x_{i}<x_{i+1}$.

ОПРЕДЕЛЕНИЕ. Построим для функции $g \in C[a, b]$ на отрезке $[c, d] \subseteq[a, b]$ расширенный альтернанс $\left\{X_{i}\right\}_{i=1}^{k}$. Альтернансным разбиением отрезка $[c, d]$ для функции $g$ будем назьвать упорядоченный набор точек $\left\{z_{i}\right\}_{i=0}^{k} \subset[c, d]$ такой, что

1) $(f-g)\left(z_{i}\right)=0 \forall i \in 1, \ldots, k-1$;

2) $z_{0}=c, z_{k}=d$

3) $X_{i}<z_{i}<X_{i+1} \forall i \in 1, \ldots, k-1$.

Через $P C\left[t_{0}, t_{1}, \ldots, t_{s}\right]$ обозначим класс кусочно постоянньг функций, заданных на отрезке $\left[t_{0}, t_{s}\right]$ и постоянных на интервалах $\left(t_{i}, t_{i+1}\right)$. Через $S_{n}\left[t_{0}, t_{1}, \ldots, t_{s}\right]$ обозначим класс полиномиальных сплайнов степени $n$ и гладкости $n-1$, заданных на отрезке $\left[t_{0}, t_{s}\right]$ и имеющих узлы в точках $t_{1}, t_{2}, \ldots, t_{s-1}$. Определение понятия сплайна можно найти, например, в [2]. Имеет место следующее соотношение: $h \in S_{n}\left[t_{0}, t_{1}, \ldots, t_{s}\right] \Longleftrightarrow h^{(n)} \in$ $P C\left[t_{0}, t_{1}, \ldots, t_{s}\right]$. В частности, из теорем $1-3$ следует, что на некотором отрезке функция $g_{*}$ будет являться сплайном из класса $S_{1}, S_{n}$ и $S_{2}$ соответственно.

Будем говорить, что функция:

- “принимает знак + или -" на множестве $X$, если существует такая точка из $X$, что функция в ней соответственно положительна или отрицательна;

- “сохраняет знак + или -" на множестве $X$, если во всех точках из $X$ функция соответственно принимает знак + или -;

- "меняет знак в точке $x \mathrm{c}-$ на $+(\mathrm{c}+$ на -$)$ ", если существует константа $\varepsilon>0$ такая, что на $(x-\varepsilon, x)$ функция сохраняет знак $-(+)$, а на $(x, x+\varepsilon)$ функция сохраняет знак $+(-)$;

- "принимает значения с чередующимися знаками на упорядоченном наборе точек $a_{1}<a_{2}<\cdots<a_{k}$ ", если она принимает в точках $a_{i}$ попеременно знаки + и - .

В дальнейшем нам также будет удобно говорить (несмотря на то, что это противоречит общепринятой терминологии), что функция “принимает знак 0" на множестве $X$, если существует такая точка из $X$, что функция в ней равна нулю.

3.2. Вспомогательные утверждения. Сначала приведем без доказательства две простые леммы. 
Лемма 1. Пусть $n \geqslant 1 u N \geqslant n+1$. Если функиия $g \in A^{n-1}$ принимает в $N$ точках $x_{1}<x_{2}<\cdots<x_{N}$ значения с чередуюшимися знаками, то найдутся точки $\left\{y_{i}\right\}_{i=1}^{N-n}$ такие, что $x_{1}<y_{1}<y_{2}<\cdots<y_{N-n}<x_{N} ;$ в них $g^{(n)}$ cуществует и принимает значения с чередующимися знаками, причем $\operatorname{sign} g^{(n)}\left(y_{1}\right)=$ $(-1)^{n} \operatorname{sign} g\left(x_{1}\right)$.

СлЕДСТВИЕ. Поскольку производная абсолютно непрерывной функиии является непрерывной в некоторой окрестности каждой точки своего существования, в условиях леммы 1 можнн утверждать не только существование точек $y_{j}$, но и существование их окрестностей, в которых $g^{(n)}$ сохраняет знак.

Лемма 2. Пусть даны функиия $f \in C[a, b] \backslash \mathscr{D}^{n}$ и функиия $g \in \mathscr{D}^{n}$. Пусть также даны отрезок $[c, d] \subseteq[a, b]$ и функиия $h \in A C^{n-1}[a, b]$ такая, что $h \equiv 0$ вне отрезка $[c, d]$, а на $[c, d]$ выполняются условия

1) $h^{(n)}$ - ограничена;

2) существует константа $\delta_{1}>0$ такая, что при $x \in O_{\delta_{1}}\left(Q_{+1}(g)\right)$ выполняется неравенство $h(x)>0$, и при $x \in O_{\delta_{1}}\left(Q_{-1}(g)\right)$ выполняется неравенство $h(x)<0$

3) существует константа $\delta_{2}>0$ такая, что в $\delta_{2}$-окрестности любой точки $x_{0}$, где выполняется равенство $\overline{\lim }_{x \rightarrow x_{0}}\left|g^{(n)}(x)\right|=1$, выполняется неравенство $\operatorname{sign} h^{(n)} \cdot \operatorname{sign} g^{(n)} \leqslant 0$.

Тогда существует такое число $\varepsilon>0$, что для функиии $g_{\varepsilon}=g+\varepsilon h$ выполнень условия $\left|g_{\varepsilon}^{(n)}\right| \leqslant\left|g^{(n)}\right| \leqslant 1,\left\|f-g_{\varepsilon}\right\|_{C[a, b]} \leqslant\|f-g\|_{C[a, b]},\left\|f-g_{\varepsilon}\right\|_{C[c, d]}<\|f-g\|_{C[c, d]}$.

Лемма 3. Пусть $n \geqslant 1$ и дан набор точек $t_{0}<t_{1}<\cdots<t_{n}$, а такжсе набор чисел $\left\{r_{i}\right\}_{i=0}^{n-1}$. Пусть выполняются равенства $\operatorname{sign} r_{i}=(-1)^{i}$ при $i=0, \ldots, n-1$. Тогда существует единственный сплайн $h \in S_{n}\left[t_{0}, t_{1}, \ldots, t_{n}\right]$, удовлетворяющий краевым условиям $h^{(i)}\left(t_{0}\right)=0 u h^{(i)}\left(t_{n}\right)=r_{i}$ nри $i \in 0, \ldots, n-1$. Более того, выполняются следующие условия:

1) $h>0$ на промежутке $\left(t_{0}, t_{n}\right]$;;

2) $h^{(n)}$ принимает на интервалах $\left(t_{i}, t_{i+1}\right)$ значения с чередующимися знаками, начиная с полохительного, т.е. $\operatorname{sign}\left(\left.h^{(n)}\right|_{\left(t_{i}, t_{i+1}\right)}\right)=(-1)^{i}$ nри $i=$ $0, \ldots, n-1$.

ДокАЗАТЕЛЬСтво. Существование и единственность сплайна $h$ следуют из общей теоремы о существовании и единственности интерполяционного сплайна (ее можно найти, например, в [2, с. 26-28]). Докажем пункты 1) и 2) леммы.

Докажем сначала, что $h \geqslant 0$ на отрезке $\left[t_{0}, t_{n}\right]$. От противного предположим, что существует точка $x_{0}$ такая, что $h\left(x_{0}\right)<0$. Поскольку $h\left(t_{0}\right)=0$ и $h\left(t_{n}\right)=r_{0}>0$, верно неравенство $t_{0}<x_{0}<t_{n}$. Это значит, что в трех последовательных точках функция $h$ принимает знаки $0,-$ и + . Применяя два раза теорему Лагранжа, мы получаем точки $x_{11}$ и $x_{12}$, в которых $h^{\prime}$ принимает знаки - и + , причем $t_{0}<x_{11}<x_{0}<x_{12}<t_{n}$. Кроме того, из краевых условий следует, что $h^{\prime}\left(t_{0}\right)=0$ и $h^{\prime}\left(t_{n}\right)=r_{1}<0$. Таким образом, мы получили четыре последовательные точки (а именно $t_{0}, x_{11}, x_{12}$ и $t_{n}$ ), в которых $h^{\prime}$ принимает знаки $0,-,+,-$. Применив три раза теорему Лагранжа, мы получим точки с чередующимися знаками уже для $h^{\prime \prime}$, и так далее. В итоге для функции $h^{(n-1)}$ найдется (с учетом краевых условий) набор из $n+2$ точек со следующими знаками: 0 , $-,+, \ldots,+(-)$. Функция $h^{(n-1)}$ есть непрерывная ломаная с конечным числом звеньев; 
значит, она абсолютно непрерьвна. Тогда по лемме 1 существует $n+1$ точка, в которых $h^{(n)}$ принимает значения с чередующимися знаками, что противоречит включению $h^{(n)} \in P C\left[t_{0}, \ldots, t_{n}\right]$. Значит, $h \geqslant 0$ на отрезке $\left[t_{0}, t_{n}\right]$.

Докажем пункт 2) леммы; пункт 1) будет доказан ниже. Поскольку $h\left(t_{0}\right)=0$ и $h\left(t_{n}\right)>0$, функция $h^{\prime}$ с учетом краевых условий принимает в трех последовательных точках знаки $0,+,-$. Точно также получаем для функции $h^{\prime \prime}$ знаки $0,+,-,+$ и так далее. В итоге мы найдем $n+1$ точку, в которых $h^{(n-1)}$ принимает знаки $0,+,-, \ldots,+(-)$. Отсюда по лемме 1 будет следовать наличие у $h^{(n)}$ ровно $n$ точек со знаками,,$+- \ldots$, $+(-)$. Учитьвая, что функция $h^{(n)} \in P C\left[t_{0}, \ldots, t_{n}\right]$, получаем требуемьй результат.

Докажем теперь пункт 1 ) леммы. От противного, пусть существует такая точка $x_{0} \neq$ $t_{0}$, что $h\left(x_{0}\right)=0$. Мы доказали, что $h^{(n)} \neq 0$, значит, функция $h$ не может иметь участков тождественного равенства нулю. Поскольку $h \geqslant 0$, то существует точка $x_{1} \in$ $\left(t_{0}, x_{0}\right)$, в которой $h\left(x_{1}\right)>0$. Это значит, что на интервале $\left(x_{1}, x_{0}\right)$ функция $h^{\prime}$ принимает знак - , а на интервале $\left(x_{0}, t_{n}\right)$ принимает знак + , кроме того, из краевых условий следует, что $h^{\prime}\left(t_{0}\right)=0$ и $h^{\prime}\left(t_{n}\right)<0$. Таким образом, мы получили четыре последовательные точки, в которых $h^{\prime}$ принимает знаки $0,-,+,-$. Продолжив рассуждения так же, как и в начале доказательства этой леммы, получим противоречие. Лемма доказана.

Имеет место и “симметричная" лемма, а именно.

Лемма 4. Пусть $n \geqslant 1$ и дан набор точек $t_{0}<t_{1}<\cdots<t_{n}$, а также набор чисел $\left\{l_{i}\right\}_{i=0}^{n-1}$. Пусть все числа $l_{i}>0$. Тогда существует единственный сплайн $h \in S_{n}\left[t_{0}, t_{1}, \ldots, t_{n}\right]$, удовлетворяюший краевым условиям $h^{(i)}\left(t_{0}\right)=l_{i} u h^{(i)}\left(t_{n}\right)=$ 0 прu $i \in 0, \ldots, n-1$.

Более того, выполняются следующие условия:

1) $h>0$ на промежутке $\left[t_{0}, t_{n}\right)$;

2) $h^{(n)}$ принимает на интервалах $\left(t_{i}, t_{i+1}\right)$ значения с чередующимися знаками, начиная с отрицательного, т.е. $\operatorname{sign}\left(\left.h^{(n)}\right|_{\left(t_{i}, t_{i+1}\right)}\right)=(-1)^{i+1}$ при $i=$ $0, \ldots, n-1$.

ДокАЗАТЕЛЬСТВО этой леммы аналогично доказательству предыдущей, кроме того, ее можно получить из леммы 3 заменой переменных.

Лемма 5. Для любих двух точек $c<d$ илюбъх чисел $y_{0}>0, y_{1}, \ldots, y_{n-1}$ существует функиия $h \in A C^{(n-1)}[c, d]$, удовлетворяющая следующим условиям:

1) $h \geqslant 0$ на отрезке $[c, d]$;

2) $h(c)=h^{\prime}(c)=\cdots=h^{(n-1)}(c)=0 u h(d)=y_{0}, h^{\prime}(d)=y_{1}, \ldots, h^{(n-1)}(d)=$ $y_{n-1}$

3) $h^{(n)}$ ограничена.

ДокАЗАТЕльСтво. Дополним список условий на функцию $h$ еще двумя:

4) $h^{(n)}(c)=1$;

5) $h^{(n)}(d)=0$.

Выделим на интервале $(c, d)$ произвольный набор из $n$ точек $a_{1}<a_{2}<\cdots<a_{n}$, и построим функцию $h_{1} \in S_{n+1}\left[c, a_{1}, \ldots, a_{n}, d\right]$, удовлетворяющую условиям 2)-5). Такой сплайн существует и единственен (см., например, [2, с. 26-28]), но может оказаться нарушенным условие 1 ). Тем не менее, поскольку $h_{1}(d)=y_{0}>0$, и первая ненулевая 
производная функции $h_{1}$ в точке $c$ больше нуля $\left(h_{1}^{(n)}(c)=1\right)$, мы можем утверждать, что существует константа $\delta>0$ такая, что на промежутках $(c, c+\delta)$ и $(d-\delta, d]$ функция $h_{1}>0$.

Известно, что для любых чисел $a_{1}<a_{2}<a_{3}<a_{4}$ существует так назьваемьй "гладкий пенек" - функция $H \in C^{\infty}$ такая, что $0 \leqslant H \leqslant 1, H \equiv 1$ на отрезке $\left[a_{2}, a_{3}\right]$, и $H \equiv 0$ вне отрезка $\left[a_{1}, a_{4}\right]$. Построим функцию $h_{2}$ - "гладкий пенек" с условиями $h_{2} \equiv 0$ на отрезках $[c, c+\delta / 3]$ и $[d-\delta / 3, d]$, и $h_{2} \equiv 2\left\|h_{1}\right\|_{C[a, b]}$ на $[c+2 \delta / 3, d-2 \delta / 3]$; $h_{2} \geqslant 0$. Очевидно, что функция $h=h_{1}+h_{2}$ удовлетворяет всем условиям леммы. Лемма доказана.

Кроме того, нам понадобится так называемая теорема Будана-Фурье для сплайнов. Полную формулировку и доказательство этой теоремы можно найти в [3]; мы приведем ее в менее общей формулировке.

ТЕОРЕМА БУДАНА-ФУРЬЕ. Пусть сплайн $h \in S_{n}\left[t_{0}, t_{1}, \ldots, t_{s}\right]$. Тогда количество нулей сплайна $h$ на отрезке $\left[t_{0}, t_{s}\right]$ с учетом их кратности не превосходит размерности пространства $S_{n}\left[t_{0}, t_{1}, \ldots, t_{s}\right]$ без единицы, т.е. $s+n-1$.

Определение кратности нуля сплайна в этой теореме обобщает понятие кратности нуля обычного полинома и совпадает с ним при отсутствии у сплайна нулей в узлах. В любом случае, нуль кратности $k$ по обычному определению будет учтен как нуль кратности не меньше $k$ по определению теоремы Будана-Фурье.

3.3. Доказательство теоремы 2. Поскольку теорема для случая $n=1$ доказана Корнейчуком, далее в доказательстве считаем, что

$$
n \geqslant 2 \text {. }
$$

Достаточность. Докажем от противного. Пусть существует функция $g$, которая удовлетворяет условиям теоремы, но не является ЭНП. Поскольку множество $\mathscr{D}^{n}$ является замкнутым и локально компактньм, хотя бы один ЭНП всегда существует (см., например, [4, с. 11]), обозначим его через $g_{*}$. Рассмотрим функцию $h=g_{*}-g$. Поскольку функция $g-$ не наилучшая, то $\left\|f-g_{*}\right\|<\|f-g\|$; значит

$$
h>0 \quad \forall x \in Q_{+1}(g) \quad \text { и } \quad h<0 \quad \forall x \in Q_{-1}(g) .
$$

Это значит, что функция $h$ принимает в точках $\left\{x_{i}\right\}_{i=1}^{k}$ чередующиеся по знаку значения; следовательно, по лемме 1 найдутся точки $\left\{y_{j}\right\}_{j=1}^{k-n}\left(t_{0}<y_{1}<y_{2}<\cdots<y_{k-n}<\right.$ $t_{s}$ ) вместе с некоторыми окрестностями, в которых функция $h^{(n)}$ принимает значения с чередующимися знаками. При этом $\operatorname{sign} h^{(n)}\left(y_{1}\right)=(-1)^{n} \operatorname{sign} h\left(x_{1}\right)$.

Предположим, что найдется такой отрезок $\left[t_{i}, t_{i+1}\right]$, на котором лежат две или более точек из построенного набора $\left\{y_{j}\right\}$. Тогда в одной из этих двух точек (а также в некоторой ее окрестности) знаки функций $g^{(n)}$ и $h^{(n)}$ совпадут. Следовательно, в этой окрестности выполнено $\left|g_{*}^{(n)}\right|=\left|g^{(n)}\right|+\left|h^{(n)}\right|=1+\left|h^{(n)}\right|>1$, т.е. $g_{*} \notin \mathscr{D}^{n}$. Таким образом, на каждом отрезке $\left[t_{i}, t_{i+1}\right]$ лежит не более одной точки из набора $\left\{y_{j}\right\}$; следовательно, верно неравенство $k-n \leqslant s$. Поскольку по п. 2) теоремы $k-n \geqslant s$, можно утверждать, что $k-n=s$ и $y_{j} \in\left(t_{j-1}, t_{j}\right)$ при $j=1, \ldots, s$. 
Поскольку точка $y_{1} \in\left(t_{0}, t_{1}\right)$, согласно п. 1$)$ теоремы верно равенство $\operatorname{sign} g^{(n)}\left(y_{1}\right)=$ $(-1)^{n} \operatorname{sign}(f-g)\left(x_{1}\right)$. С другой стороны, по (4) верно равенство $\operatorname{sign}(f-g)\left(x_{1}\right)=$ $\operatorname{sign} h\left(x_{1}\right)$; значит,

$$
\operatorname{sign} g^{(n)}\left(y_{1}\right)=(-1)^{n} \operatorname{sign}(f-g)\left(x_{1}\right)=(-1)^{n} \operatorname{sign} h\left(x_{1}\right)=\operatorname{sign} h^{(n)}\left(y_{1}\right) .
$$

Таким образом, в точке $y_{1} \in\left(t_{0}, t_{1}\right)$ знаки функций $g^{(n)}$ и $h^{(n)}$ совпали, что противоречит включению $g_{*} \in \mathscr{D}^{n}$. Достаточность доказана.

Необходимость. Пусть дан элемент наилучшего приближения $g_{*}$; необходимо проверить для него вьполнение всех условий теоремы. Далее под уклонением мы будем понимать величину $\left\|f-g_{*}\right\|$. Доказательство мы разобьем на несколько этапов.

Этап 1. Определим множество $M$ как

$$
M=\left\{x \in[a, b]: \varlimsup_{y \rightarrow x} g_{*}^{(n)}(y)=1 \text { или } \underline{\lim }_{y \rightarrow x} g_{*}^{(n)}(y)=-1\right\} .
$$

Обозначим $\bar{M}=[a, b] \backslash M$. Поскольку множество $M$ замкнутое, на любом отрезке $[u, v] \subset \bar{M}$ выполнено $\left\|g_{*}^{(n)}\right\|_{C[u, v]}<1-r([u, v])$ для некоторой константы $r([u, v])>$ 0, зависящей от $[u, v]$.

Выберем произвольньй непустой отрезок $[c, d] \subset[a, b]$. Выделим рядом с ним (если $a<c)$ непустой отрезок $\left[c_{1}, c\right]$. Аналогично выделим непустой отрезок $\left[d, d_{1}\right]$. Предположим, что $\left[c_{1}, d_{1}\right]$ - это “+"-отрезок, а отрезки $\left[c_{1}, c\right]$ и $\left[d, d_{1}\right]$ принадлежат $\bar{M}$.

На отрезке $[c, d]$ определим функцию $h(x)=-g_{*}(x)+A$. Тогда на отрезке $[c, d]$ выполнится равенство $h^{(n)}=-g_{*}^{(n)}$. Кроме того, увеличивая константу $A$ в определении $h$ можно добиться выполнения неравенства $h>0$ на отрезке $[c, d]$. По лемме 5 можно построить положительную функцию $h_{1} \in A C^{n-1}\left[c_{1}, c\right]$ такую, что $h_{1}^{(i)}\left(c_{1}\right)=0$ и $h_{1}^{(i)}(c)=h^{(i)}(c)$ для $i \in 0, \ldots, n-1$. Пусть функция $h(x) \equiv 0$ при $x<c_{1}, h \equiv h_{1}$ на отрезке $\left[c_{1}, c\right]$. Аналогично продолжим функцию $h$ справа от точки $d$.

В результате мы получили функцию $h$, удовлетворяющую лемме 2 на отрезке $\left[c_{1}, d_{1}\right]$ и равную нулю вне его; следовательно, существует функция $g_{\varepsilon}>0$ такая, что $\| f-$ $g_{\varepsilon}\|\leqslant\| f-g_{*} \|$ и $g_{\varepsilon} \in \mathscr{D}^{n}$. При этом вьполняется неравенство $\left\|f-g_{\varepsilon}\right\|_{C[c, d]}<\| f-$ $g_{*} \|_{C[c, d]}$.

Таким образом, если есть некоторьй отрезок $[c, d]$, к которому примыкает интервал $\left(c_{1}, c\right) \subset \bar{M}$ (или $\left.c=a\right)$ и примыкает $\left(d, d_{1}\right) \subset \bar{M}$ (или $\left.d=b\right)$, причем $\left(c_{1}, d_{1}\right)$ является "+"- или "-"-интервалом, то можно построить такую поправку $\varepsilon h$ к функции $g_{*}$, которая в целом на $[a, b]$ не увеличивает уклонение и не выводит $g_{*}$ за пределы $\mathscr{D}^{n}$, но локально на $[c, d]$ уменьшает уклонение и удаляет точки из $M$.

Значит, можно считать, что множество $M$

1) не пусто, так как иначе при помощи описанной процедуры легко уменьшить уклонение на всем отрезке, что противоречит наилучшести $g_{*}$;

2 ) состоит только из отрезков длиной не менее чем $\gamma / 4$ (см. (2)), так как более короткие отрезки и изолированные точки будут заведомо “+”- или “-"-отрезками, и иметь примькающие интервалы, лежащие в $\bar{M}$;

3) каждый из отрезков, найденных в предыдущем пункте, есть замькание набора интервалов, на которых $g_{*}^{(n)} \equiv 1$ или $g_{*}^{(n)} \equiv-1$. От противного, пусть есть отрезок $[c, d] \subset M$, не удовлетворяющий этому высказыванию. Это значит, что на нем найдется интервал $(u, v)$ такой, что внутри него нет ни одного интервала постоянства $g_{*}^{(n)}$. 
Поскольку производная непрерьвна в некоторой окрестности каждой точки своего существования, можно сделать вывод, что на интервале $(u, v)$ функция $g_{*}^{(n)}$ не существует, что противоречит включению $g_{*} \in \mathscr{D}^{n}$.

Этап 2. Назовем участком пилообразности набор смежных интервалов, на которых поочередно $g_{*}^{(n)} \equiv 1$ или $g_{*}^{(n)} \equiv-1$. Друг от друга интервалы отделены точками разрыва функции $g_{*}^{(n)}$. Под мощностью участка пилообразности мы будем понимать количество смежных интервалов, из которых он состоит.

Выше мы доказали, что если функция $g_{*}-$ ЭНП, то существует хотя бы один участок пилообразности, по крайней мере, из одного интервала. Кроме того, можно считать, что все множество $M$ есть объединение конечного числа участков пилообразности, которые, впрочем, могут состоять из бесконечного числа смежных интервалов. На любом интервале внутри участкапилообразности точки разрьва $g_{*}^{(n)}$ не могут быть всюду плотны (так как тогда $g_{*}^{(n)}$ на этом интервале не существует), но они могут иметь точки сгущения. Точки сгущения разрьвов также не могут быть плотны на любом интервале. Это значит, что в любой достаточно малой окрестности любой точки сгущения нет других точек сгущения и, следовательно, есть (по крайней мере, с одной стороны от нее) участок пилообразности из произвольного конечного количества интервалов.

Пусть имеется некоторьй отрезок $[u, v] \subset[a, b]$ такой, что к нему примькают два участка пилообразности: $\left\{\left(u_{i-1}, u_{i}\right)\right\}_{i=-n-1}^{0}$ и $\left\{\left(v_{j}, v_{j+1}\right)\right\}_{j=0}^{n+1}$, причем $u_{0}=u$ и $v_{0}=$ $v$, а отрезок $\left[u_{-n-1}, v_{n+1}\right]$ является “+”-отрезком. Определим на отрезке $[u, v]$ функцию

$$
h(x)=-g_{*}(x)+A+B \cdot\left(x-\frac{u+v}{2}\right)^{n-1} .
$$

Видно, что выполняется равенство $h^{(n)}=-g_{*}^{(n)}$. Кроме того, увеличивая $A$, можно добиться того, чтобы вьполнилось неравенство $h>0$ на отрезке $[u, v]$, а увеличивая $B$, можно добиться еще и вьполнения равенств $\operatorname{sign} h^{(i)}(u)=(-1)^{i}$ и $h^{(i)}(v)>0$ при $i \in$ $0, \ldots, n-1$.

Построим сплайн $h_{1} \in S_{n}\left[u_{-n}, u_{-n+1}, \ldots, u_{0}\right]$, определяемый следующими краевыми условиями: при $i \in 0, \ldots, n-1$ вьполняются равенства $h_{1}^{(i)}\left(u_{-n}\right)=0$ и $h_{1}^{(i)}\left(u_{0}\right)=$ $h^{(i)}\left(u_{0}\right)$. По лемме 3 такой сплайн существует. Также построим сплайн $h_{2} \in S_{n}\left[v_{0}\right.$, $\left.v_{1}, \ldots, v_{n}\right]$ с краевыми условиями $h_{2}^{(i)}\left(v_{n}\right)=0$ и $h_{2}^{(i)}\left(v_{0}\right)=h^{(i)}\left(v_{0}\right)$ при $i \in 0, \ldots, n-1$. Этот сплайн существует по лемме 4 . Пусть функция $h$ на отрезке $\left[u_{-n}, u_{0}\right]$ равна функции $h_{1}$, а на отрезке $\left[v_{0}, v_{n}\right]-$ функции $h_{2}$. Если окажется, что на отрезке $\left[u_{-n}, u_{0}\right]$ выполнено $\operatorname{sign} g_{*}^{(n)}=\operatorname{sign} h^{(n)}$, то необходимо присоединить к $[u, v]$ интервал $\left(u_{1}, u_{0}\right)$ и повторить все рассуждения на отрезке $\left[u_{-n-1}, u_{1}\right]$. Мы снова попадем в условия леммы 3 , но на этот раз получим sign $g_{*}^{(n)}=-\operatorname{sign} h^{(n)}$. При необходимости аналогично можно поступить и на отрезке $\left[v_{0}, v_{n+1}\right]$. Продолжим функцию $h$ на оставшийся отрезок $[a, b]$ тождественным нулем. Получили функцию $h$, удовлетворяющую условиям леммы 2 на отрезке $\left[u_{-n}, v_{n}\right]$; значит, существует функция $g_{\varepsilon} \in \mathscr{D}^{n}$ такая, что на отрезке $\left[u_{-n}, v_{n}\right]$ нет точек из множеств $Q_{+1}\left(g_{\varepsilon}\right)$ и $Q_{-1}\left(g_{\varepsilon}\right)$ и участков пилообразности функции $g_{\varepsilon}$. При этом неважно, сколько точек сгущения лежало на отрезке $[u, v]$.

Таким образом, мы можем считать, что если участок пилообразности является "+" или “-"-отрезком и на нем можно выделить по $n+1$ смежному интервалу с каждого конца, то он состоит не более чем из $2 n+3$ интервалов. Следовательно, можно считать, 
что у нас нет точек сгушения, к которьп точки разрывов сгущаются с двух сторон, поскольку достаточно малая окрестность самой точки сгущения будет заведомо "+"- или “-"-отрезком, а также будет иметь по набору из $n+1$ интервалов с каждого конща. Кроме того, мы можем считать, что у односторонних точек сгущения со стороны сгущения участок пилообразности сколь угодно короткий, а далее есть интервал, на котором $\left|g_{*}^{(n)}\right|<1-\varepsilon$ для некоторого $\varepsilon>0$ и нет точек максимального уклонения. Таким образом, можно считать, что есть только участки пилообразности конечной мощности, возможно с примькающими к ним на краях точками сгущения.

Эman 3. Рассмотрим произвольный максимальньй по включению участок пилообразности конечной мощности. Покажем, что если на нем нет жесткого звена, то можно построить поправку к $g_{*}$, которая на этом участке одновременно уменьшит уклонение и уменьшит модуль $g_{*}^{(n)}$, тем самым мы избавимся от этого участка пилообразности. Действуя таким образом, мы или найдем жесткое звено, или покажем, что $g_{*}$ не является ЭНП.

Предположим, что на рассматриваемом участке пилообразности нет жесткого звена. По определению он состоит из $s$ смежных интервалов $\left\{\left(t_{i}, t_{i+1}\right)\right\}_{i=0}^{s-1}$. Из максимальности по включению следует, что либо существует константа $\delta>0$ такая, что на интервале $\left(t_{0}-\delta, t_{0}\right)$ вьполнено неравенство $\left|g_{*}^{(n)}(x)\right|<1$, либо $t_{0}$ - односторонняя точка сгущения (разрывы сгущаются слева). В обоих случаях мы можем считать, что при достаточно малых $\varepsilon>0$ на интервале $\left(t_{0}-2 \varepsilon, t_{0}-\varepsilon\right)$ нет точек максимального уклонения и вьполнено неравенство $\left|g_{*}^{(n)}\right| \leqslant 1-\varepsilon_{1}$ для какой-то константы $\varepsilon_{1}>0$. Аналогично можно считать, что на интервале $\left(t_{s}+\varepsilon, t_{s}+2 \varepsilon\right)$ выполнено неравенство $\left|g_{*}^{(n)}\right| \leqslant 1-\varepsilon_{1}$. Построим на $\left[t_{0}, t_{s}\right]$ функцию $h$ такую, что

$$
h>0 \text { на } Q_{+1}\left(g_{*}\right) \quad \text { и } h<0 \text { на } Q_{-1}\left(g_{*}\right),
$$

и, кроме того,

$$
\operatorname{sign} h^{(n)}=-\operatorname{sign} g_{*}^{(n)} .
$$

Продолжим построенную функцию $h$ влево от точки $t_{0}$ с гладкостью $n-1$ полиномом степени $n-1$ до точки $t_{0}-\varepsilon$. Поскольку число $\varepsilon$ сколь угодно мало, можно считать, что выполнено равенство $\operatorname{sign}\left(\left.h\right|_{\left(t_{0}-\varepsilon, t_{0}\right)}\right)=\operatorname{sign} h\left(t_{0}\right)$. Далее продолжим функцию $h$ на интервал $\left(t_{0}-2 \varepsilon, t_{0}-\varepsilon\right)$ по лемме 5 так, чтобы выполнились условия $h^{(i)}\left(t_{0}-2 \varepsilon\right)=0$ при $i \in 0, \ldots, n-1$. Совершенно аналогично поступим и справа от точки $t_{s}$. Пусть $h \equiv 0$ вне отрезка $\left[t_{0}-2 \varepsilon, t_{s}+2 \varepsilon\right]$. Получим функцию $h$, удовлетворяющую лемме 2 . Следовательно, имея такую функцию $h$, мы сможем избавиться от участка пилообразности $\left[t_{0}, t_{s}\right]$ вне зависимости от возможного наличия точек сгушения на его концах.

Таким образом, для доказательства теоремы нам осталось показать, что мы можем построить функцию $h$ с условиями (5) и (6). При этом поведение этой функции вне отрезка $\left[t_{0}, t_{s}\right]$ можно не учитывать, мы всегда сможем ее продолжить за пределы отрезка $\left[t_{0}, t_{s}\right]$ описанным выше образом.

Мы будем строить функцию $h$ в виде сплайна из класса $S_{n}\left[t_{0}, t_{1}, \ldots, t_{s}\right]$. Ниже мы наложим на $h$ интерполящионные условия, обеспечивающие выполнение требования (5). Эти условия будут строиться на основе альтернансного разбиения отрезка $\left[t_{0}, t_{s}\right]$. Если количество точек в разбиении будет мальм, то условий будет недостаточно для однозначного определения сплайна, в этом случае нам понадобятся дополнительные условия. Удобнее всего получить недостающие условия, добавляя в альтернанс “фиктивные 
точки". При построении интерполяционных условий мы не будем различать настоящие и фиктивные точки альтернанса. Поскольку будем только добавлять условия, сплайн, построенный с учетом новых условий, будет удовлетворять нашим начальным требованиям. Кроме того, мы будем расширять отрезок $\left[t_{0}, t_{s}\right]$, т.е. увеличивать область определения сплайна, что тоже никак не скажется на вьполнении требований (5) и (6) на исходном отрезке $\left[t_{0}, t_{s}\right]$. Мы покажем ниже, что наши интерполяционные условия обеспечат и вьполнение условия (6). С учетом всех этих оговорок, приступим к построению функции $h$.

Построим на отрезке $\left[t_{0}, t_{s}\right]$ расширенньй альтернанс $\left\{X_{i}\right\}_{i=1}^{k}$. Введем обозначение: $S_{i}=\operatorname{sign}\left(\left.\left(f-g_{*}\right)\right|_{X_{i}}\right)$. Имеет место следующее очевидное соотношение $S_{i}=$ $(-1)^{i+1} S_{1}$. Поскольку $\left[t_{0}, t_{s}\right]$ - участок пилообразности, можно ввести следующее обозначение:

$G_{i}=\left.g_{*}^{(n)}\right|_{\left(t_{i}, t_{i+1}\right)}$. Также имеет место соотношение $G_{i}=(-1)^{i} G_{0}$.

Обозначим через $D_{i, j}$ разность между количеством выпуклых оболочек множеств расширенного альтернанса $\overline{X_{i}}$, пересекаюшихся с $\left[t_{i}, t_{j}\right]$, и числом $j-i$. Имеет место следуюшее свойство символа $D_{i, j}$ : пусть $p<q<r$; тогда

$$
D_{p, r}=D_{p, q}+D_{q, r}-\delta(q)
$$

где $\delta(q)=1$, если $t_{q} \in \overline{X_{l}}$ для некоторого $l$, и $\delta(q)=0$ в противном случае.

Через $W_{i}$ будем обозначать номер первого из множеств расширенного альтернанса, пересекающихся с отрезком $\left[t_{i}, t_{s}\right]$. Пусть $w=W_{i}$; поскольку на любом отрезке $\left[t_{i}, t_{j}\right]$ нет жесткого звена, то

$$
\begin{array}{ll}
D_{i, j} \leqslant n-1, & \text { если } G_{i}=(-1)^{n} S_{w}, \\
D_{i, j} \leqslant n, & \text { если } G_{i}=-(-1)^{n} S_{w} .
\end{array}
$$

Это значит, что

$$
D_{i, j} \leqslant n \quad \forall 0 \leqslant i<j \leqslant s .
$$

Применив условия (8) к отрезку $\left[t_{0}, t_{s}\right]$, получаем неравенства

$$
\begin{array}{ll}
D_{0, s} \leqslant n-1, & \text { если } G_{0}=(-1)^{n} S_{1}, \\
D_{0, s} \leqslant n, & \text { если } G_{0}=-(-1)^{n} S_{1} .
\end{array}
$$

Без ограничения общности можно считать, что

$$
G_{0}=-(-1)^{n} S_{1}
$$

В самом деле, если это не так, то мы можем добавить фиктивную точку альтернанса $x_{0}=t_{0}-1$ со знаком уклонения $-S_{1}$, и принять за новьй $\left(t_{0}, t_{1}\right)$ интервал $\left(x_{0}, t_{1}\right)$, считая, что $\left.\left.g_{*}^{(n)}\right|_{\left(x_{0}, t_{0}\right)} \equiv g_{*}^{(n)}\right|_{\left(t_{0}, t_{1}\right)}$. Ясно, что если у нас до добавления не было жесткого звена, то оно и не появилось, так как добавленная точка не может попасть в требуемьй теоремой альтернанс из-за условия пункта 1 теоремы. В дальнейшем будем считать, что точки альтернанса пронумерованы с 1 до $k$ и выполнено условие (10).

Рассмотрим три возможных случая: $D_{0, s}=n, D_{0, s}=n-1$ и $D_{0, s} \leqslant n-2$. 
а) Случай $D_{0, s}=n$. Построим на $\left[t_{0}, t_{s}\right]$ альтернансное разбиение $\left\{z_{i}\right\}_{i=0}^{k}$. Введем набор точек $\left\{y_{i}\right\}_{i=0}^{k}$ и положим $y_{i}=z_{i}$. Это значит, что верны неравенства

$$
X_{i}<y_{i}<X_{i+1} \quad \text { при } i \in 1, \ldots, k-1 .
$$

Наложим на $h$ следующие $k$ интерполяционных условий: $h\left(y_{0}\right)=S_{1}, h\left(y_{i}\right)=0$ при $i \in 1, \ldots, k-1$. Необходимое и достаточное условие существования и единственности такого интерполящионного сплайна заключается (см., например, [2, с. 26-28]) в выполнении равенства $k=s+n$ и в справедливости неравенств $y_{i-1}<t_{i}<y_{i+n}$ при $i \in 1, \ldots, s-1$. Вообще говоря, для исходного набора $\left\{y_{i}\right\}_{i=0}^{k}$ эти неравенства могут и не выполниться. Покажем, что его можно изменить так, что неравенства вьполнятся при сохранении (11). Пусть не выполнилось неравенство $t_{i}<y_{i+n}$. Поскольку $D_{0, i} \leqslant n$, верно неравенство $t_{i}<X_{i+n+1}$. Следовательно, точку $y_{i+n}$ можно выбрать так, что вьполнятся неравенства $t_{i}<y_{i+n}<X_{i+n+1}$. Пусть не вьполнилось неравенство $y_{i-1}<t_{i}$. Поскольку $D_{i, s} \leqslant n$, то $D_{0, i}=D_{0, s}-D_{i, s}+\delta(i) \geqslant n-n+\delta(i) \geqslant 0$; значит, верно неравенство $X_{i-1}<t_{i}$, и можно выбрать точку $y_{i-1}$ так, чтобы вьполнились неравенства $X_{i-1}<y_{i-1}<t_{i}$. Заметим, что если исходная точка $y_{i}$ не удовлетворяла левому неравенству (т.е. $y_{i} \geqslant t_{i+1}$ ), то она заведомо удовлетворяла правому неравенству (т.е. $y_{i} \geqslant t_{i-n}$ ) и наоборот. Это значит, что описанная процедура выбора точек $\left\{y_{i}\right\}$ корректна.

Значит, сплайн $h$, определяемьй такими условиями, существует и единственен. По теореме Будана-Фурье для сплайнов (см. [3, с. 161]) количество нулей сплайна $h$ с учетом кратности не превосходит $k-1$. Следовательно, кроме точек $\left\{y_{i}\right\}_{i=1}^{k-1}$, сплайн $h$ не имеет других нулей, и все они простые. Кроме того, не существует интервалов, на которых $h \equiv 0$. По построению $h\left(t_{0}\right)=S_{1}$, функция $h$ меняет знак только в точках $y_{i}$ между множествами $X_{i}$; следовательно, условие (5) выполнилось.

Мы получили, что функция $h$ принимает значения с чередующимися знаками в $k$ точках альтернанса. По лемме 1 найдется упорядоченный набор из $k-n=s$ точек $\left\{u_{j}\right\}_{j=1}^{s}$, в которых функция $h^{(n)}$ принимает значения с чередующимися знаками, причем по лемме 1 и соотношению (10) получаем $\operatorname{sign} h^{(n)}\left(u_{1}\right)=(-1)^{n} \operatorname{sign}\left(\left.h\right|_{X_{1}}\right)=(-1)^{n} S_{1}=-G_{0}$. Поскольку обе функции $h^{(n)}$ и $g_{*}^{(n)}$ лежат в классе $P C\left[t_{0}, t_{1}, \ldots, t_{s}\right]$, условие $(6)$ также выполнилось и, значит, в случае а) утверждение теоремы доказано.

b) Случай $D_{0, s}=n-1$. Добавим фиктивную точку альтернанса $x_{k+1}=t_{s}+1$ c соответствующим знаком уклонения. Так же, как и раньше, расширим интервал $\left(t_{s-1}, t_{s}\right)$ вправо до $\left(t_{s-1}, x_{k+1}\right)$. Покажем, что при таком добавлении не возникло жесткого звена. В самом деле, пусть жесткое звено возникает на отрезке $\left[t_{i}, t_{s}\right]$. Пусть $l=W_{i}$; тогда число множеств $X_{j}$, пересекавшихся с отрезком $\left[t_{i}, t_{s}\right]$ до добавления, было равно $D_{0, s}+s-l+1=s+n-l$; значит, до добавления выполнялось равенство $D_{i, s}=s+$ $n-l-(s-i)=i+n-l$. Поскольку на отрезке $\left[t_{i}, t_{s}\right]$ жесткого звена не было, но при добавлении одной точки справа оно возникло, то до добавления вьполнялось одно из равенств

i) $D_{i, s}=n-1$, если $G_{i}=(-1)^{n} \cdot S_{l}$,

ii) $D_{i, s}=n$, если $G_{i}=-(-1)^{n} \cdot S_{l}$.

В случае і) выполняется равенство $l=i+1$; следовательно,

$$
G_{0}=(-1)^{i} G_{i}=(-1)^{i+n} S_{l}=(-1)^{i+n+(l+1)} S_{1}=(-1)^{2 i+n+2} S_{1}=(-1)^{n} S_{1} .
$$


В случае іi) мы имеем равенство $i=l$; следовательно,

$$
G_{0}=(-1)^{i} G_{i}=(-1)^{i+n+1} S_{l}=(-1)^{i+n+1+(l+1)} S_{1}=(-1)^{2 i+n+2} S_{1}=(-1)^{n} S_{1} .
$$

В обоих случаях получили $G_{0}=(-1)^{n} S_{1}$, что противоречит условию (10).

Это значит, что описанное вьше добавление сводит случай b) к случаю а). Итак, в случае b) утверждение теоремы также доказано.

c) Случай $D_{0, s} \leqslant n-2$. Будем добавлять слева и справа фиктивные точки альтернанса с соответствующими знаками уклонения, расширяя отрезок $\left[t_{0}, t_{s}\right]$ по мере надобности за счет удлинения крайних интервалов. При этом заметим, что если мы смогли добавить слева нечетное количество фиктивных точек, то сможем добавить еще одну; следовательно, по-прежнему будет выполнено условие (10).

В итоге мы либо получим один из двух рассмотренных вьше случаев, либо возникнет ситуация, когда при добавлении дополнительной точки слева или справа возникает жесткое звено, но все равно вьполнено неравенство

$$
D_{0, s} \leqslant n-2
$$

Положим

$$
p=\max \left\{i: D_{0, i} \geqslant n-1\right\}, \quad q=\min \left\{i: D_{i, s} \geqslant n-1\right\} .
$$

Множество таких $i$ в обоих определениях не пусто, так как и слева, и справа нельзя добавить фиктивную точку альтернанса без возникновения жесткого звена. Из неравенства (12) следует, что $1 \leqslant p, q \leqslant s-1$.

Согласно $(9)$ и (13) вьполняется неравенство $n-1 \leqslant D_{0, p} \leqslant n$. Покажем, что $D_{0, p}=$ $n-1$. От противного, пусть $D_{0, p}=n$. По определению (13) вьполняется неравенство

$$
D_{0, p+1} \leqslant n-2 \text {. }
$$

По свойству (7) имеем $D_{0, p+1}=D_{0, p}+D_{p, p+1}-\delta(p)$, а по определению $D_{i, j}$ вьполняется неравенство $D_{p, p+1} \geqslant \delta(p)-1$; следовательно, $D_{0, p+1} \geqslant D_{0, p}-1=n-1$, что противоречит (14). Установили равенство

$$
D_{0, p}=n-1
$$

Покажем, что $q \geqslant p$. От противного, предположим, что $q<p$; тогда согласно $(12)$

$$
D_{0, s}=\left(D_{0, q}+D_{q, p}+D_{p, s}-\delta(q)-\delta(p)\right) \leqslant n-2
$$

Кроме того, по (7) и (13) получаем

$$
D_{q, p}+D_{p, s}-\delta(p)=D_{q, s} \geqslant n-1 \quad \text { и } \quad D_{0, q}+D_{q, p}-\delta(q)=D_{0, p}=n-1 .
$$

Сложение двух этих неравенств дает

$$
D_{q, p} \geqslant 2 n-2-\left(D_{0, q}+D_{q, p}+D_{p, s}-\delta(q)-\delta(p)\right) .
$$


Применяя (16), получаем неравенство $D_{q, p} \geqslant n$; тогда из (9) следует, что $D_{q, p}=n$. Пусть $w=W_{q}$. Из равенства $D_{q, p}=n$ и неравенств (8) для отрезка $\left[t_{q}, t_{p}\right]$ следует, что

$$
G_{q}=-(-1)^{n} S_{w}
$$

Подставляя равенство $D_{q, p}=n$ во второе из равенств (17), получаем равенство $D_{0, q}=-1+\delta(q)$. С другой стороны, по определению $D_{0, q}=(w-1)-q+\delta(q)$; значит, выполняется равенство $w=q$. Применяя (18), получаем следующее равенство:

$$
G_{0}=(-1)^{q} G_{q}=(-1)^{n+q+1} S_{w}=(-1)^{n+q+w} S_{1}=(-1)^{n} S_{1},
$$

что противоречит условию (10).

Доказали, что $p \leqslant q$. Равенство $p=q$ такженевозможно, так как в этом случае будет иметь место неравенство $D_{0, s} \geqslant D_{0, p}+D_{p, s}-1 \geqslant 2 n-3$. Учитьвая (3), получаем, что $D_{0, s} \geqslant 2 n-3 \geqslant n-1$, что противоречит (12). Следовательно, верно неравенство $p<q$.

Введем следующие обозначения:

$$
l=\max \left\{i: X_{i} \text { пересекается c }\left[t_{0}, t_{p}\right]\right\}, \quad r=\max \left\{i:\left[t_{0}, t_{i}\right) \text { не пересекается с } X_{l+1}\right\} \text {. }
$$

Очевидно, что $p \leqslant r$. Заметим, что $l=D_{0, p}+p$, что с учетом (15) дает равенство $l=p+n-1$. Поскольку $p \geqslant 1$ и $n \geqslant 2$, то $l \geqslant 2$.

Далее рассмотрим два подслучая:

1) $r \geqslant l$

2) $r<l$.

1) Подслучай $r \geqslant l$. Это неравенство означает, что на $\left[t_{p}, t_{l}\right)$ нет точек из $X_{l+1}$. В этом случае мы построим функцию $h \in S_{n}\left[t_{0}, t_{1}, \ldots, t_{l}\right]$ и продолжим ее при помощи тождественно равной нулю функции на $\left[t_{l}, t_{s}\right]$. Потребуем, чтобы $h$ удовлетворяла условиям (5) и (6) только на промежутке $\left[t_{0}, t_{l}\right)$. Такая функция $h$ позволит уменьшить уклонение не на всем отрезке $\left[t_{0}, t_{s}\right]$, а только на $\left[t_{0}, t_{l}\right)$. Кроме того, она избавит нас от участка пилообразности $\left[t_{0}, t_{l}\right]$. В результате мы вернемся на начало этапа с отрезком $\left[t_{l}, t_{s}\right]$ в качестве нового $\left[t_{0}, t_{s}\right]$. Поскольку $l \geqslant 2$, то в нем будет меньше интервалов $\left(t_{i}, t_{i+1}\right)$, чем в $\left[t_{0}, t_{s}\right]$.

Построим на отрезке $\left[t_{0}, t_{l}\right]$ альтернансное разбиение $\left\{z_{i}\right\}_{i=0}^{l}$. Введем в рассмотрение набор точек $\left\{y_{i}\right\}_{i=0}^{l}$ и первоначально положим $y_{i}=z_{i}$. Потребуем от $h$ выполнения следуюших интерполяционных условий:

1) $h\left(y_{0}\right)=S_{1}, h\left(y_{i}\right)=0$ при $i \in 1, \ldots, l-1$;

$2)$ в точке $t_{l}$ функция $h$ должна с гладкостью $n-1$ сопрягаться с нулевой функцией, т.е. $h\left(t_{l}\right)=h^{\prime}\left(t_{l}\right)=\cdots=h^{(n-1)}\left(t_{l}\right)=0$.

Необходимыми и достаточньми условиями существования и единственности интерполящионного сплайна с краевыми условиями являются (см. [4, с. 85]):

i) равенство количества условий интерполяции и числа свободных параметров;

ii) вьполнение неравенств $d_{i} \geqslant i+1$, при $i \in 0, \ldots, n+1$, где $d_{i}$ - есть количество условий, наложенных на сплайн и его производные вплоть до порядка $i$;

iii) вьполнение неравенств $k_{a i} \geqslant i$ и $k_{b i} \geqslant l-i$, где $k_{a i}$ и $k_{b i}$ есть количество всех условий, наложенных на сплайн и все его производные на промежутках $\left[t_{0}, t_{i}\right)$ и $\left(t_{i}, t_{l}\right]$ соответственно. 
Покажем, что эти условия выполняются.

i) Число условий интерполящии и число свободных параметров равно $l+n$.

ii) Имеется $l+1$ интерполяционное условие на сам сплайн и по одному условию на каждую его производную вплоть до порядка $n-1$. Это значит, что при $i \leqslant n-1$ вьполняется $d_{i}=l+1+i \geqslant i+1$. Учитывая, что $l \geqslant 2$, получаем оставшиеся неравенства $d_{n}=l+n \geqslant n+1$ и $d_{n+1}=l+n \geqslant n+2$.

iii) Установим первое неравенство. От противного, пусть для какого-то $i$ вьполнено $k_{a i}<i$, т.е. $y_{i}>t_{i}$. Покажем, что мы можем выбрать точку $y_{i}$ так, что вьполнится $X_{i}<y_{i}<t_{i}$. В самом деле, это невозможно, только если множество $\overline{X_{i}}$ пересекается с отрезком $\left[t_{i}, t_{p}\right]$. Это означает, что

$$
D_{i, p} \geqslant(l-(i-1))-(p-i)=n .
$$

Из неравенства (9) следует, что $D_{i, p}=n$, а это с учетом (8) влечет равенство $G_{i}=$ $-(-1)^{n} S_{i}$, и тогда

$$
G_{0}=(-1)^{i} G_{i}=(-1)^{n+1+i} S_{i}=(-1)^{n+1+i+(i-1)} S_{1}=(-1)^{n} S_{1},
$$

что противоречит условию (10). Таким образом, мы всегда можем выбрать точку $y_{i}$ между $X_{i}$ и $X_{i+1}$ так, что неравенство $k_{a i} \geqslant i$ выполнится.

Установим второе неравенство. При $i \geqslant p$ будет $l-i \leqslant n-1$, при этом $k_{b i}=n$, так как в точке $t_{l}$ имеется ровно $n$ условий. Поэтому осталось установить неравенство только при $i<p$. Допустим противное, что $k_{b i}<l-i$ для некоторого $i$, т.е. число условий интерполяции на отрезке $\left[t_{i}, t_{l}\right]$ строго меньше $l-i$. В точке $t_{l}$ наложено $n$ условий интерполяции; на промежутке $\left(t_{p}, t_{l}\right]$ других условий нет; следовательно, на отрезке $\left[t_{i}, t_{p}\right]$ условий строго меньше, чем $l-i-n$. На отрезке $\left[t_{0}, t_{p}\right]$ имеется ровно $l$ условий; значит, на отрезке $\left[t_{0}, t_{i}\right]$ их строго больше, чем $l-(l-i-n)=i+n$, т.е. $D_{0, i}>n$, что невозможно по (9).

Таким образом, такой сплайн $h$ существует и единственен. По теореме Будана-Фурье для сплайнов других нулей, кроме точек $\left\{y_{i}\right\}_{i=1}^{l}$, у сплайна $h$ нет. По построению точка $t_{l}$ есть нуль кратности $n$; следовательно, все остальные нули $\left\{y_{i}\right\}_{i=1}^{l-1}$ простые, в них сплайн меняет знак. Это значит, что на отрезке $\left[t_{0}, t_{l}\right]$ условие $(5)$ вьполнилось.

Покажем теперь, что на отрезке $\left[t_{0}, t_{l}\right]$ условие $(6)$ также выполнилось. Доказательство этого факта похоже на доказательство леммы 3 , поэтому приведем его схематично. Мы знаем, что сплайн $h$ принимает на упорядоченном наборе из $l+1$ точки (альтернанс и точка $t_{l}$ ) знаки $S_{1},-S_{1}, \ldots, \pm S_{1}, 0$. Следовательно, $h^{\prime}$ (с учетом краевого условия) принимает на другом упорядоченном наборе из $l+1$ точки значения со знаками $-S_{1}, S_{1}, \ldots, \mp S_{1}, 0$. Продолжая этот процесс, мы убедимся, что $h^{(n-1)}$ в $(l+1)$ точке принимает значения со знаками $(-1)^{n-1} S_{1},-(-1)^{n-1} S_{1}, \ldots, \pm(-1)^{n-1} S_{1}, 0$. Таким образом, функция $h^{(n)}$ в $l$ точках принимает значения со знаками $(-1)^{n} S_{1},-(-1)^{n} S_{1}$, $\ldots, \mp(-1)^{n} S_{1}$. Учитывая (10), получаем

$$
\operatorname{sign}\left(\left.h^{(n)}\right|_{\left(t_{0}, t_{1}\right)}\right)=(-1)^{n} S_{1}=-G_{0}=-\operatorname{sign}\left(\left.g_{*}^{(n)}\right|_{\left(t_{0}, t_{1}\right)}\right) .
$$

Поскольку $h^{(n)} \in P C\left[t_{0}, \ldots, t_{l}\right]$, на отрезке $\left[t_{0}, t_{l}\right]$ условие $(6)$ также вьполнилось.

$2)$ Подслучай $r<l$. В этом случае мы добавим специальным образом внутрь отрезка $\left[t_{0}, t_{s}\right]$ две фиктивные точки альтернанса и покажем, что при этом не возникнет 
жесткого звена. После этого надо будет вернуться на начало этапа, при этом $k$ увеличится на 2 , а $s$ останется без изменений.

По построению, вьполнено неравенство $p \leqslant r$, на $\left[t_{0}, t_{r}\right)$ нет точек из $X_{l+1}$, а также, по предположению,

$$
r<l
$$

Опишем алгоритм добавления пары фиктивных точек альтернанса. Мы расположим их между множествами $X_{l}$ и $X_{l+1}$ с соответствуюшими знаками уклонения. Если $X_{l} \subset$ $\left[t_{0}, t_{p}\right)$, то добавим две фиктивные точки альтернанса в промежуток $\left(t_{p}, t_{p+1}\right)$, иначе добавим их строго между $X_{l}$ и следующей точкой $t_{i}$. Видно, что в любом случае они попадут внутрь одного интервала $\left(t_{i}, t_{i+1}\right)$, причем $i \geqslant p$.

Покажем, что добавление этих двух точек по описанному алгоритму не вызовет появления жесткого звена. В самом деле, пусть возникло жесткое звено на отрезке $\left[t_{u}, t_{v}\right]$. Поскольку обе добавленные точки альтернанса лежат на отрезке $\left[t_{u}, t_{v}\right]$, то $p<v$, что с учетом (13) дает неравенство

$$
D_{0, v} \leqslant n-2 .
$$

Покажем, что $u \geqslant p$. Предположим, что $u<p$; тогда получаем следующую цепочку неравенств: $0 \leqslant u<p<v$. Из (7) и (15) следует равенство

$$
n-1=D_{0, p}=D_{0, u}+D_{u, p}-\delta(u),
$$

поэтому

$$
D_{u, p}=(n-1)-D_{0, u}+\delta(u) .
$$

По свойству (7) и неравенству (20) получаем неравенство $D_{0, v}=D_{0, u}+D_{u, v}-$ $\delta(u) \leqslant n-2$, откуда $D_{0, u} \leqslant(n-2)+\delta(u)-D_{u, v}$. Подставляя это неравенство в $(22)$, получаем неравенство

$$
D_{u, p} \geqslant D_{u, v}+1
$$

Пусть $w=W_{u}$. Возникновение жесткого звена при добавлении этих двух точек означает, что до добавления выполнялось одно из двух неравенств:

$$
\begin{aligned}
& \text { i) } \quad D_{u, v} \geqslant n-2, \quad \text { если } G_{u}=(-1)^{n} S_{w}, \\
& \text { ii) } D_{u, v} \geqslant n-1, \quad \text { если } G_{u}=-(-1)^{n} S_{w} .
\end{aligned}
$$

В случае і) вьполняется неравенство $D_{u, v} \geqslant n-2$; подставляя его в $(23)$, получаем неравенство $D_{u, p} \geqslant n-1$. Поскольку $G_{u}=(-1)^{n} S_{w}$, и на отрезке $\left[t_{u}, t_{p}\right]$ нет жесткого звена, из (8) получаем $D_{u, p} \leqslant n-1$. Значит, $D_{u, p}=n-1$. Подставляя это равенство в $(21)$, получаем равенство

$$
D_{0, u}=\delta(u)
$$

Поскольку $X_{w}$ - первое из множеств, пересекающееся с $\left[t_{u}, t_{s}\right]$, то $\delta(u)=1$, только если $X_{w}$ пересекается и с $\left[t_{0}, t_{u}\right]$. Следовательно, равенство $(25)$ означает, что $w-1=u$, и мы получаем

$$
G_{0}=(-1)^{u} G_{u}=(-1)^{n+u} S_{w}=(-1)^{n+u+w-1} S_{1}=(-1)^{n+u+u} S_{1}=(-1)^{n} S_{1},
$$

что противоречит условию (10). 
Аналогично, в случае іi) вьполняется неравенство $D_{u, v} \geqslant n-1$. Подставляя его в $(23)$, получаем $D_{u, p} \geqslant n$. Тогда из (9) следует, что $D_{u, p}=n$. Подставляя это равенство в $(21)$, будем иметь $D_{0, u}=\delta(u)-1$. Из этого равенства получаем, что $w-1=u-1$. Следовательно,

$$
G_{0}=(-1)^{u} G_{u}=-(-1)^{n+u} S_{w}=-(-1)^{n+u+w-1} S_{1}=-(-1)^{n+u+u-1} S_{1}=(-1)^{n} S_{1},
$$

что противоречит условию (10). Следовательно, $p \leqslant u$.

Покажем, что $p<u$. Предположим противное; пусть $u=p$. Рассмотрим два случая:

i) $\delta(p)=0$;

ii) $\delta(p)=1$.

В случае і) $X_{l}$ не пересекается с $\left[t_{p}, t_{v}\right]$; следовательно, с учетом (3) и (24) получаем соотношение $D_{0, v}=D_{0, p}+D_{p, v} \geqslant n-1+n-2=2 n-3 \geqslant n-1$, которое противоречит (20).

В случае іi) множество $X_{l}$ является первым из множеств расширенного альтернанса, которые пересекаются с $\left[t_{p}, t_{v}\right]$; следовательно,

$$
D_{0, v}=D_{0, p}+D_{p, v}-1 \geqslant(n-1)+(n-2)-1=2 n-4
$$

Применение $(20)$ дает $2 n-4 \leqslant n-2$, т.е. $n \leqslant 2$. Сопоставив последнее неравенство с (3), получаем, что в этом случае $n=2$.

Поскольку $l=p+n-1$, то

$$
G_{p}=(-1)^{p} G_{0}=-(-1)^{n+p} S_{1}=-(-1)^{n+p+p+n} S_{l}=-S_{l} .
$$

Подставляем $n=2$ в (24); с учетом (26) будем иметь неравенство $D_{p, v} \geqslant n-1$. Отсюда устанавливаем следующее неравенство:

$$
D_{0, v}=D_{0, p}+D_{p, v}-1 \geqslant(n-1)+(n-1)-1=2 n-3 \geqslant n-1,
$$

что противоречит (20).

Таким образом, в любом случае верно неравенство $p<u$.

По алгоритму добавления точек выполнено неравенство $u \leqslant r$, т.е. верны следующие неравенства: $p<u \leqslant r$. Это значит, что на $\left(t_{p}, t_{u}\right)$ не может быть других множеств альтернанса, кроме $X_{l}$; отсюда $D_{0, u}=l-u=(p+n-1)-u=(n-1)-(u-p)$. По построению добавленные точки лежат правее $t_{u}$. Учитывая неравенство $u \geqslant p+1$, можно утверждать, что добавленные точки лежат правее $t_{p+1}$. По алгоритму добавления точек получаем, что $X_{l}$ пересекается с $\left[t_{u}, t_{v}\right]$, т.е. $\delta(u)=1$. Таким образом, справедливы равенства

$$
D_{0, v}=D_{0, u}+D_{u, v}-1=(n-1)-(u-p)+D_{u, v}-1 .
$$

В сочетании с (20) получаем неравенство

$$
u-p \geqslant D_{u, v}
$$

В силу (24) в любом случае имеет место неравенство $D_{u, v} \geqslant n-2$; отсюда по (27) следует, что $u-p \geqslant n-2$, т.е. $u \geqslant p+n-2=l-1$.

Рассмотрим два случая: 
i) $u<r$;

ii) $u=r$.

В случае і) получаем $r>u \geqslant l-1$, т.е. $r \geqslant l$, что противоречит (19).

В случае іi) справедливо неравенство $r \geqslant l-1$. По (19) имеет место неравенство $r<l$, поэтому $u=r=l-1$. Отсюда получаем, что

$$
G_{l-1}=(-1)^{l-1} G_{0}=(-1)^{n+l} S_{1}=(-1)^{n+l+l-1} S_{l}=-(-1)^{n} S_{l} .
$$

Поскольку $X_{l}$ - первое из множеств расширенного альтернанса, пересекающихся с отрезком $\left[t_{u}, t_{v}\right]$, в формуле $(24)$ надо использовать $w=l$. Следовательно, по формуле $(24)$ и с учетом равенства $G_{l-1}=-(-1)^{n} S_{l}$ устанавливаем неравенство $D_{u, v} \geqslant n-1$. Подставляя его в (27), получаем неравенство $u-p \geqslant n-1$. Итак, имеет место неравенство $r-p \geqslant n-1$, и, значит, $r \geqslant p+n-1=l$, что противоречит (19).

Таким образом, предположив, что при описанном добавлении двух фиктивных точек альтернанса возникает жесткое звено, мы пришли к противоречию. Подслучай 2) случая с) полностью рассмотрен.

Теорема доказана.

Автор благодарит В. И. Бердьшева за постановку задачи и С. Н. Васильева за помощь в работе над статьей.

\section{СПИСОК ЦИТИРОВАННОЙ ЛИТЕРАТУРЫ}

[1] Корнейчук Н. П. Экстремальные задачи теории приближения. М.: Наука, 1976.

[2] Завьялов Ю. С., Квасов Б. И., Мирошниченко В. М. Методы сплайн функций. М.: Наука, 1980.

[3] Larry L. Schumaker Spline functions: basic theory. New York, 1981.

[4] Корнейчук Н. П. Точные константы в теории приближения. М.: Наука, 1987. 